\title{
Appointment Lead Time Policy Development to Improve Patient Access to Care
}

Yu-Li Huang1; Sarah M. Bach²

${ }^{1}$ Mayo Clinic, College of Medicine, Rochester, Minnesota, United States; ${ }^{2}$ University of Chicago Medical Center, Center for Quality, Chicago, Illinois, United States

\section{Keywords}

Appointment, lead time, scheduling, patient access, policy

\section{Summary}

Background: Patient access to care has been a known and continuing struggle for many health care providers. In spite of appointment lead time policies set by government or clinics, the problem persists. Justification for how lead time policies are determined is lacking.

Objectives: This paper proposed a data-driven approach for how to best set feasible appointment target lead times given a clinic's capacity and appointment requests.

Methods: The proposed approach reallocates patient visits to minimize the deviation between actual appointment lead time and a feasible target lead time. A step-by-step algorithm was presented and demonstrated for return visit (RV) and new patient (NP) types from a Pediatric clinic excluding planned visits such as well-child exam and the same day urgent appointments. The steps are: $1 . \mathrm{Ob}$ tain appointment requests; 2 . Initialize a target lead time; 3 . Set up an initial schedule; 4 . Check the feasibility based on appointment availability; 5 . Adjust schedule backward to fill appointment slots earlier than the target; 6 . Adjust schedule forward for appointments not able to be scheduled earlier or on target to the later slots; 7 . Trial different target lead times until the difference between earlier and later lead time is minimized.

Results: The results indicated a $59 \%$ lead time reduction for RVs and a $45 \%$ reduction for NPs. The lead time variation was reduced by $75 \%$ for both patient types. Additionally, the opportunity for the participating clinic to achieve their organization's goal of a two-week lead time for RVs and a twoday lead time for NPs is discussed by adjusting capacity to increase one slot for NP and reduce one slot for RV.

Conclusions: The proposed approach and study findings may help clinics identify feasible appointment lead times.

\section{Correspondence to:}

\section{Dr. Yu-Li Huang}

Mayo Clinic, College of Medicine,

Rochester, Minnesota

United States

Email: huang.yuli@mayo.edu
Appl Clin Inform 2016; 7: 954-968

http://dx.doi.org/10.4338/ACl-2016-03-RA-0044

received: March 28, 2016

accepted: September 10, 2016

published: October 19, 2016

Citation: Huang Y, Bach SM. Appointment lead time policy development to improve patient access to care. Appl Clin Inform 2016; 7: 954-968

http://dx.doi.org/10.4338/ACl-2016-03-RA-0044 


\section{Background}

Timely access is a key component of high quality healthcare. This goal of improving healthcare access has become ubiquitous throughout the industry. To achieve this goal countries with national healthcare systems have implemented scheduling targets mandating acceptable lead times until an appointment. Canada's provincial governments have mandated a 4 week maximum lead time for radiation therapy treatment for cancer [1]. Sweden's lead time guarantee assures access to a general practitioner within 7 days and a specialist within 90 days [2]. The UK's National Health Service (NHS) mandates a maximum 18 week lead time to see a specialist, 2 week if cancer is suspected, and a 48 hour for genitourinary medicine [3-5]. In the US, the Institute of Medicine recently released a report focused on transforming access and scheduling. This report recommends future research into standardized measures and benchmarks for timely access to health care as national standards do not currently exist [6].

A traditional scheduling approach involves reserving a fixed number of daily appointment slots for different patient types, such as reserving slots for urgent patients requiring same day appointments $[7,8]$. To balance the access needs of differing patient acuity levels clinics have studied the optimal number of appointment slots to keep open for same day appointments [9]. Additional scheduling research has focused on defining appointment slot lengths for different patient types and service times [10-12] and designing improved scheduling templates [13-15]. Factors such as the order of appointment types, appointment lengths, and where add on appointments are placed throughout a schedule have been shown to significantly impact schedule performance and clinic throughput [16]. However, this traditional approach can lead to poor performance if appointments are scheduled far in advance. Long appointment lead times have been shown to increase the probability of patient no show which leads to overall decreased clinic throughput [17]. Research has focused on how to predict no shows and cancellations $[18,19]$ and design optimal overbooking policies to mitigate the effect of no shows $[20,21]$. Therefore, the alternative scheduling approach gaining popularity is open access, also known as advanced access, where a majority of the clinic day is not scheduled in advance and patients call in for same or next day appointments [22]. Yet, barriers exist to implementing open access such as the increased burden placed on patients to call in for appointments as they cannot schedule in advance [23]. Additionally, many clinics are unprepared to handle the challenge of matching provider capacity to variable daily demand which can lead to longer lead times overall [24]. Specifically, the NHS cited this issue with matching variable demand and provider capacity as a barrier [25]. Other approaches, such as short booking windows have been studied and shown to perform better compared to open access while still providing patients with timely access to care [26].

In spite of the numerous efforts to improve scheduling methods, the issue of patient access to care persists. Outside of government mandated lead times, many clinics have tried to set their own policies around target lead times. One possible motivation for selecting target lead times could be due to quality goals defined by hospitals, such as to reduce the current waiting time within quality improvement programs. These policies may be clinically based such as with chemotherapy regimens that indicate specific timeframes within treatment must occur [27]. However, most clinics have flexibility in how they set these target lead time policies; examples show a variety in selected target wait times from a gastroenterology clinic's goal of seeing new patients within 10 days [28], the Military Health System's goal of seeing routine return primary care visits within 1 week [6], a urology clinic's goal of seeing new patients within 5 days [29], and an academic medical center's goal of seeing patients in the correct specialty within 3 days [30]. It is difficult to discern a clinic's motivation for selecting a specific target lead time. Some seem to have been set from the stand point of clinical safety by medical providers [31], while others attribute these decisions to their desire to incorporate patients' preferences [32]. Most patients prefer shorter lead times, especially when seeking general practitioner appointments [33]; however, some survey studies conclude that lead time tolerance can range up to two weeks [34]. Studies also indicate that patient waiting longer than expected lead times results in dissatisfaction [35]. Others conclude that lead time preferences depend on patient characteristics such as age, gender, social status, health conditions, and illness and other factors, such as the continuity of care with one doctor or choice of appointment time during the day, are often more important to patients than days until appointment [36, 37]. To the best of our know- 
ledge, there is a lack of study evaluating lead time policy performance and the effects lead time policy selection has on appointment outcomes and scheduling.

\section{Objectives}

This paper explores how to best set appointment target lead times for each patient type, excluding planned visits such as well child exam and the same day urgent appointments, given a clinic's capacity and appointment requests. The proposed approach reallocates patient visits to minimize the deviation between actual appointment lead time and target lead time.

\section{Methods}

The study aims to determine the feasible lead time policy of each patient type for the unplanned and non-urgent visits using an algorithm that minimizes the total lead time from appointment requests given appointment availability. The approach is summarized as follows: 1. Obtain appointment requests; 2 . Initialize a target lead time; 3 . Set up an initial schedule by scheduling all requested appointments at the target lead time; 4 . Check the feasibility based on appointment availability; 5 . Adjust schedule backward to fill appointment slots earlier than the target; 6. Adjust schedule forward for appointments not able to be scheduled earlier or on target to the later slots; 7 . Trial different target lead time until the difference between earlier and later lead time is minimized. In the other words, the target lead time is selected to minimize the difference. The incremental lead time is calculated by the absolute value of the difference between total lead time of backward and forward adjustments, abs $\left(L_{b}-L_{f}\right)$. Steps 1-6 of the approach are demonstrated through a hypothetical example in a 6 -day period. A case study from a pediatric clinic is presented for step 7. The following notations are used throughout the paper.

$R(i)=$ the total appointment request at day $i$.

$t=$ the target lead time.

$C(i)=$ appointment capacity or availability at day $i$.

$E_{j}(i)=$ excess capacity at day $i$, where $j$ is schedule adjustment for $t$ (target lead time), $b$ (moving backward), and $f$ (moving forward). $E_{t}(i)$ is the excess capacity after schedule adjustment when target lead time $t$ is set. $E_{b}(i)$ is the excess capacity after backward schedule adjustment and $E_{f}(i)$ is the excess capacity after forward schedule adjustment.

$S_{k}(i)=$ appointment schedule at day $i$ adjusted based on situation $k$, where $k=t$ (target lead time), $b$ (moving backward), and $f$ (moving forward).

$L_{b}=$ total lead time generated by backward adjustment.

$L_{f}=$ total lead time generated by forward adjustment.

\subsection{Appointment request}

Most scheduling systems capture when appointments are created. In this study, appointment created date was used to determine the amount of daily appointment requests. For this example, assume for day $1,2,3,4,5$ and 6 , the incoming number of appointment requests are 5, 6, 3, 7, 2, and 9, respectively, hence, $R(1)=5, R(2)=6, R(3)=3, R(4)=7, R(5)=2$, and $R(6)=9$; see $>$ Table 1 .

\subsection{Target lead time and initial schedule}

The target lead time can be set at any value. In our example, the target lead time is set to be 3 days ( $t$ $=3$ ), which means that the five patients requesting appointments on day one should be scheduled no later than day four where $S_{t}(i+t)=R(i)$. The algorithm starts by initially scheduling all requested appointments at the target lead time. Hence, by incorporating the target lead time, the appointment requests for day $1,2,3,4,5$ and 6 should be initially scheduled at day $4,5,6,7,8$, and 9 , where $S_{t}(4)=$ $5, S_{t}(5)=6, S_{t}(6)=3, S_{t}(7)=7, S_{t}(8)=2$, and $S_{t}(9)=9$; $>$ Table 1 . 


\subsection{Appointment feasibility}

After the initial schedule is created, a feasibility check is needed to see if the clinic's current capacity is sufficient for the requested appointments. The capacity can be fixed or varied daily. In this example, we assume a capacity, or appointment availability, of four every day, $C(i)=4$ where $i=1,2$, $\ldots, 12$. The excess capacity from the initial schedule, $E(i)$, is calculated by $C(i)-S_{t}(i)$. In this example, $E_{t}(4)=C(4)-S_{t}(4)=4-5=-1, E_{t}(5)=-2, E(6)=1, E_{t}(7)=-3, E_{t}(8)=2$, and $E_{t}(9)=-5$; see $>$ Table $1 . E_{t}(i)<0$ indicates the initial schedule based on target lead time $t$ at day $i$ exceeds the capacity at day $i$.

\subsection{Backward schedule adjustment}

When $E_{t}(i)<0$, the excess patients need to be scheduled to a day other than day $i$. It is preferred to search appointment openings prior to the target lead time until the request day in order for the clinic to honor the lead time policy. Therefore, the search starts from $i-1$ to $i-t$. The adjustment will start from the latest day, day 9 . The search algorithm is the following:

Step 1: initialize $E_{b}(i)$, let $E_{b}(i)=E_{t}(i)$

Step 2: if $E_{b}(i)<0$, search backward until $E_{b}(i-n)>0$, where $n=1,2, \ldots, t$.

Step 3: modify $\left(E_{b}(i), S_{b}(i)\right)$ and $\left(E_{b}(i-n), S_{b}(i-n)\right)$

Step 4: repeat step 2 and 3 until $E_{b}(i-t)<0$

In Table $1 E_{t}(i=9)=-5$ which means five patients need to be moved from day 9 , the search starts from $\mathrm{i}-1=9-1=8$ to $i-t=9-3=6 . E_{t}(8)=2$ means there are two appointment slots available, so two of the five patients from day 9 are moved to day $8 . E_{t}(7)=-3$ means there are no spots available. $E_{t}(6)=1$ means one of the remaining three patients can be moved to day 6 . Then, the backward search ends. The remaining two patients stay at day 9. From this schedule adjustment the following updates are seen in Table $1\left(E_{b}(9), S_{b}(9)\right)=(-2,6),\left(E_{b}(8), S_{b}(8)\right)=(0,4)$, and $\left(E_{b}(6), S_{b}(6)\right)=(0,4)$. Next, we check $E_{t}(8)$; however, since $E_{t}(8)=0$ there is no capacity issue. Next, $E_{b}(7)=-3$, which means three patients need to be moved from day 7 , the search starts from day 6 to day 4 . $E_{b}(6)$ is now 0 meaning that there is no appointment spot available. $E_{b}(5)=-2$ and $E_{b}(4)=-1$ means there are no spots available, as well. Then, the backward search ends. All three excess capacity patients at day 7 stay at day 7 and there is no update needed. Next, we check $E_{t}(6)$; however, since $E_{t}(6)=0$ there is no capacity issue. $E_{b}(5)=-2$ which means two patients need to be moved from day 5 . The backward search starts from day 4 to day 2 . Since $E_{b}(3)=4$, both patients can be moved to day 3 . Then, the search ends. The updates are $\left(E_{b}(5), S_{b}(5)\right)=(0,4)$ and $\left(E_{b}(3), S_{b}(3)\right)=(2,2)$. Lastly, $E_{b}(4)=-1$ indicates one patient needs to be moved. Since $E_{b}(3)=2$ this patient can be moved to day 3 . Therefore, this patient is moved to day 3 and the updates are $\left(E_{b}(4), S_{b}(4)\right)=(0,4)$ and $\left(E_{b}(3), S_{b}(3)\right)=(1$, $3)$.

\subsection{Forward schedule adjustment}

Patients who are scheduled on days where the capacity is exceeded even after the backward schedule adjustment will be scheduled forward from their current scheduled day. The forward search starts at the earliest day where the capacity is exceeded, day 7 in the example. There is no limit how far forward the search can go since patients need to be scheduled somewhere. The search algorithm is similar to the backward search and outlined as follows:

Step 1: initialize $E_{f}(i)$, let $E_{f}(i)=E_{b}(i)$

Step 2: if $E_{f}(i)<0$, search forward until $E_{f}(i+n)>0$, where $n=1,2, \ldots, m$. $m$ can be as far in the future as the schedule allows.

Step 3: modify $\left(E_{f}(i), S_{f}(i)\right)$ and $\left(E_{f}(i+n), S_{f}(i+n)\right)$

Step 4: repeat step 2 and 3 until all $E_{f}(i)>0$

$\checkmark$ Table 1 shows that the first $E_{f}(i)<0$ is $E_{f}(7)=-3$. Looking forward, the first possible opening is at day 10 where $E_{f}(10)=4$. Hence, these three patients are moved to day 10 . The updates are $\left(E_{f}(7)\right.$, $\left.S_{f}(7)\right)=(0,4)$ and $\left(E_{f}(10), S_{f}(10)\right)=(1,3)$. The next $E_{f}(i)<0$ occurs at day 9 where $E_{f}(9)=-2$. Day 10 has one slot available $\left(E_{f}(10)=1\right)$ and day 11 has four spots available $\left(E_{f}(11)=4\right)$. Therefore, one pa- 
tient is moved to day 10 and another is moved to day 11 . The updates are $\left(E_{f}(9), S_{f}(9)\right)=(0,4)$, $\left(E_{f}(10), S_{f}(10)\right)=(0,4)$, and $\left(E_{f}(11), S_{f}(11)\right)=(3,1)$.

\subsection{Lead time measurement}

The study goal is to determine the lead time policy for each patient type. The proposed method focuses on measuring the lead time generated by backward and forward adjustments. The example in - Table 1 summarized the total lead time generated by backward adjustment $\left(L_{b}\right)$ is 10 days and by forward adjustment $\left(L_{f}\right)$ is 12 days. Backward adjustments include two patients moved from day 9 to day 8 , one patient from day 9 to day 6 , two patients from day 5 to day 3 , and one patient from day 4 to day 3 . The total lead time is then calaculated by 2 (patient) $\times 1$ (day) $+1 \times 3+2 \times 2+1 \times 1=10$ days. The calculation is similar for forward adjustments. There are three patients moved from day 7 to day 10 , one patient from day 9 to day 10 , and one patient from day 9 to day 11 . The total lead time is calaculated by $3 \times 3+1 \times 1+1 \times 2=12$ days.

\subsection{Incremental lead time optimization}

The backward adjustment actually reduces lead time, while the forward adjustment increases lead time. If the initial target lead time is longer, set further ahead, there are more chances for backward adjustment. On the other hand, if the target lead time is shorter, set closer to the appointment request day, the chances of forward adjustment are higher. The decision then is how to set the target lead time value $(t)$ in order to minimize the incremental lead time. The incremental lead time is calculated by the absolute value of the difference between total lead time of backward and forward adjustments, $\operatorname{abs}\left(L_{b}-L_{f}\right)$. It represents the changes of these adjustments from the target lead time. The key is to ensure the overall lead time after adjustments is closest to the target lead time. Therefore, the goal is to minimize abs $\left(L_{b}-L_{f}\right)$ by changing the target lead time $(t)$. The optimization is demonstrated through a case study for the return visit patient in a Pediatric clinic. The optimization steps are: 1.

Step 1: Select a target lead time - The initial target lead time $(t)$ could start from one day, $t=1$.

Step 2: Find the total lead time for backward $\left(L_{b}\right)$ and forward $\left(L_{f}\right)$ adjustment given $t$.

Step 3: Calculate the absolute value of the lead time difference, abs $\left(L_{b}-L_{f}\right)$, between backward $\left(L_{b}\right)$ and forward $\left(L_{f}\right)$ adjustment given $t$.

Step 4: Repeat Step 1 to Step 3 for $t+1$.

Step 5: Compare abs $\left(L_{b}-L_{f}\right)$ given $t$ and $t+1$. If abs $\left(L_{b}-L_{f}\right)$ given $t<\operatorname{abs}\left(L_{b}-L_{f}\right)$ given $t+1$, then $\operatorname{abs}\left(L_{b}-L_{f}\right)$ given $t$ is the minimum and the optimization search stops. Otherwise, set abs $\left(L_{b}-L_{f}\right)$ given $t+1$ to be minimum and repeat step 1 to 4 for $t+1$.

\subsection{Case study}

This case study is based on six months of data from a Pediatric clinic including appointment date and time, appointment type, and appointment created date and time. The lead time is calculated by the difference between the appointment date and the appointment created date. Patients are scheduled on a scheduling template either by as first come first serve or by their acuity. The clinic has four different appointment types: new patient (NP), return visit (RV), well child exam (WCE), and the same day appointment. The distribution percentages of these appointment types are $3.8 \%, 22.4 \%$, $26.7 \%$, and $47.1 \%$, respectively. The lead time policy decision does not affect WCE appointments, which are planned at regularly scheduled intervals far in the future, or same day appointments, which are used for unplanned urgent cases and are required to be seen right away. However, the clinic does have discretion on setting a lead time policy for NP and RV patient types. NP appointments are scheduled for 30 minutes and RV appointments for 15 minutes. On any given clinic day, there are two physicians seeing about 50 total patients. Hence, the daily capacity for the return visit patients is about $50 \times 22.4 \% \approx 11$ appointment slots and for new patients is about $50 \times 3.8 \% \approx 2$ appointment slots. The proposed algorithm was used to set the feasible target lead time starting from one day. $>$ Figure 1 indicates that the incremental lead time was minimized when the target lead 
time was four or five days for RV and three days for NP. The minimized absolute difference of incremental lead time between forward and backward adjustment was 80 days for RV and 4 days for NP.

When the RV target lead time is equal to four days, the total lead time from the backward adjustment is 323 days and from the forward adjustment is 243 days. When RV target lead time is equal to five days, the total lead time from the backward adjustment is 327 days and from the forward adjustment is 247 days. In this case, the clinic can choose either one option. For NP where the target lead time is three days, the total lead time from the backward adjustment is 57 days and from the forward adjustment is 61 days. The target lead time should be set at three days in order to optimize the incremental lead time.

\section{Results}

\subsection{The feasible target lead time for current patient types}

The case study for return visit patients in the Pediatric clinic concluded the feasible target lead time for RV patients was either 4 or 5 days. The proposed method reallocated the appointment schedule for 6 months (128 days) of requests given a fixed appointment capacity of 11 slots in a clinic day. To have a fair comparison to the current lead time, we analyzed results for a variable capacity model as well. The variable capacity, unlike the fixed capacity set at $11 \mathrm{RV}$ slots in our example, varies appointment capacity daily. There are many reasons a clinic may have variable capacity of RV appointment slots such as patient preference, sudden changes of appointment schedule due to physicians' schedule, or weather conditions. Hence, the actual number of RV patients scheduled for the same time period was used as the variable capacity. Using the proposed method with the varied capacity, the target RV lead time was minimized at 7 days. The results indicated that the current lead time for return visit patients is 9.4 days on average with a standard deviation of 6.4 days. The proposed method with fixed capacity reduces the lead time to 3.9 days on average with a standard deviation of 1.7 days when target lead time is 4 days, which is $59 \%$ reduction on average and reduces 4.7 days variation from the current lead time. As for target lead time of 5 days, the average lead time is 4.9 days and the standard deviation of 1.5 days, which reduces $48 \%$ on average and about 5 days variation from the current lead time. The proposed method with variable capacity has the average lead time of 7.1 days and a standard deviation of 5.6 days, which is $25 \%$ reduction on average and about one day variation reduction from the current lead time. $>$ Figure 2 shows lead time distribution for current, proposed with fixed capacity at the target of 4, proposed with fixed capacity at the target of 5 , and proposed with varied capacity at the target of 7 . $>$ Figure 2 also demonstrates visually how much lead time variation was reduced and the target lead time was shifted to fewer days using the proposed algorithm on RV patients.

As for new patients, the study concluded the feasible target lead time was 3 days given a fixed appointment capacity of 2 slots in a clinic day. The actual number of NP patients scheduled for the same time period was also used as the variable capacity for a fair comparison to the current lead time. Using the proposed method with the varied capacity, the target lead time was minimized at 5 days. The results indicated that the current lead time for new patients is 5.5 days on average with a standard deviation of 8.0 days. The proposed method with fixed capacity reduces the lead time to 3.0 days on average with a standard deviation of 2.0 days, which is $45 \%$ reduction on average and reduces 6 days on variation from the current lead time. The proposed method with variable capacity has the average lead time of 5.0 days and a standard deviation of 3.1 days, which is $8 \%$ reduction on average and about 5 days reduction on variation from the current lead time. $>$ Figure 3 shows NP lead time distribution for current, proposed with fixed capacity at the target of 3 , and proposed with varied capacity at the target of 5 . $>$ Figure 3 again demonstrated visually on the reduction of lead time variation and the target lead time for NP patients. For both RV and NP cases, the proposed method reduces not only the average lead time, but also the spread of the lead time. 


\subsection{The impact of appointment capacity to target lead time}

In this section, the impact of changes to appointment capacity to achieve a desired lead time target policy is discussed. If a clinic desires to institute a certain lead time target they may need to adjust appointment capacities to do so effectively. Continuing with the RV patient case, the appointment capacity is currently at 11 patient slots in a clinic day, which generates the target lead time policy to be either 4 or 5 days. If the clinic wishes to reduce the lead time for RVs to be less than 2 days on average, the appointment capacity has to be increased to 12 slots in a clinic day and the lead time policy set to be 2 days; Table 2. In the case of NPs, the current capacity is 2 appointment slots in a clinic day, which has a target lead time of 3 days. If the clinic prefers to have all new patients scheduled within 2 days on average, the capacity needs to increase to 3 slots in a clinic day. Table 2 summarized the changes on target lead time, lead time from backward and forward adjustment, the minimum absolute difference, and average lead time. A common preference among clinics is to have a two-week lead time for return visit patients and a two-day lead time for new patients. For this Pediatric clinic to achieve this goal the capacity for NP needs to be increased to 3 slots in a day, which requires an additional slot. For this clinic, an NP slot is scheduled for 30 minutes and a RV slot is scheduled for 15 minutes. To accommodate an additional NP slot, the clinic needs to reassign two RV slots, which reduces the number of RV slots to 9 slots. $>$ Table 2 indicates that the average lead time at capacity of 9 would increase significantly from 4.9 days to 29.2 days. However, if there is no difference on slot length or duration between RV and NP, reassigning one RV slot to NP slot would only increase average lead time to 14.1 days, which is roughly two weeks. $>$ Figure 4 shows the tradeoff of the target lead time when switching between NP and RV capacity where the duration of NP slot is twice of RV slot and where the duration of NP slot is the same as RV slot; Figure 4 . This result demonstrates an example where in order to achieve a target lead time defined by the government or organization, an adjustment of capacity is necessary. If the capacity cannot be adjusted from other patient types, the alternatives will be to increase work hours or hire additional medical providers.

Instead of using a scheduling template with a fixed capacity for each patient type, an alternative solution to the capacity planning is to allow capacity to be varied due to demand variation on different appointment types. Clinics often adjust capacity by adding one type and reducing another appointment type on certain days. In our example, clinic days could vary between having two or three appointment slots for NP and adjust RV capacity accordingly. However, the proposed algorithm assumes the demand and the capacity are known when calculating the target lead time. A further investigation is needed to examine or even predict the pattern of appointment requests so that the capacity can be planned accordingly. In the case where NP slots are filled and a new patient needs to be place in a RV slot without running the algorithm for capacity changes, this new patient will have the target lead time of RVs.

\section{Discussion}

As literature stated, most patients prefer shorter lead times, but about $17 \%$ of patients can tolerate longer lead times of up to two weeks for unplanned, non-urgent visits. One study found that patients' willingness to wait was impacted by certain patient characteristics. Specifically, beyond the expected lead time the study found patients who prefer to choose their own doctors and times were willing to wait one extra day, female patients were willing to wait two extra days, and elderly patients were willing to wait two and half extra days [36]. Overall, the two primary concerns of patients are: 1. Shortening appointment lead times; 2 . Reducing the variation of actual lead time from the expected lead time. The proposed approach, as demonstrated through the case study, indicates the achievement of accommodating both concerns. Clinics should place patients in available appointment slots according to patients' preference when they can, but should negotiate within patients' lead time tolerance. The results indicated an as much as 59\% lead time reduction from the current 9.4 days to the proposed 3.9 days for return visit patients and a $45 \%$ reduction from 5.5 days to 3 days for new patients. The lead time variation also reduced $77 \%$ for return visit patients and $75 \%$ for new patients. This result achieved patients' preferences of shorter lead time and lead time variation. 
The results also discussed the impact of capacity changes on appointment lead time and demonstrated how to adjust capacity needed for each patient type in order to achieve a target appointment lead time defined by a law or organization. Limitations include the need to provide: 1 . Systematic guidelines for incorporating patient scheduling preferences to place patients in the most appropriate appointment slots; 2. Reasons for appointment changes and appointment demand pattern for better capacity planning; 3 . Feasibility of the proposed algorithm to be automated in currently scheduling systems. These limitations require further investigation and could be potential future research.

\section{Conclusions}

This paper proposed a data driven approach to define the appointment lead time policy based on appointment requests and clinic capacity. A step-by-step algorithm was presented and demonstrated for return visit and new patient types from a Pediatric clinic. The proposed approach will not only assist clinics in setting appointment lead times, but will also consequently reduce lead time to potentially increase patient access to care.

\section{Clinical Relevance Statement}

This study aimed to provide a better appointment lead time policy by setting feasible target lead times based on a clinic's capacity and appointment requests. The step-by-step approach discussed using a real-life case in a Primary Care clinic could guide clinics to establish their lead time policy.

\section{Conflict of Interest}

The authors declare that they have no conflict of interest.

\section{Protection of Human and Animal Subjects}

The research study was not human subject research. 


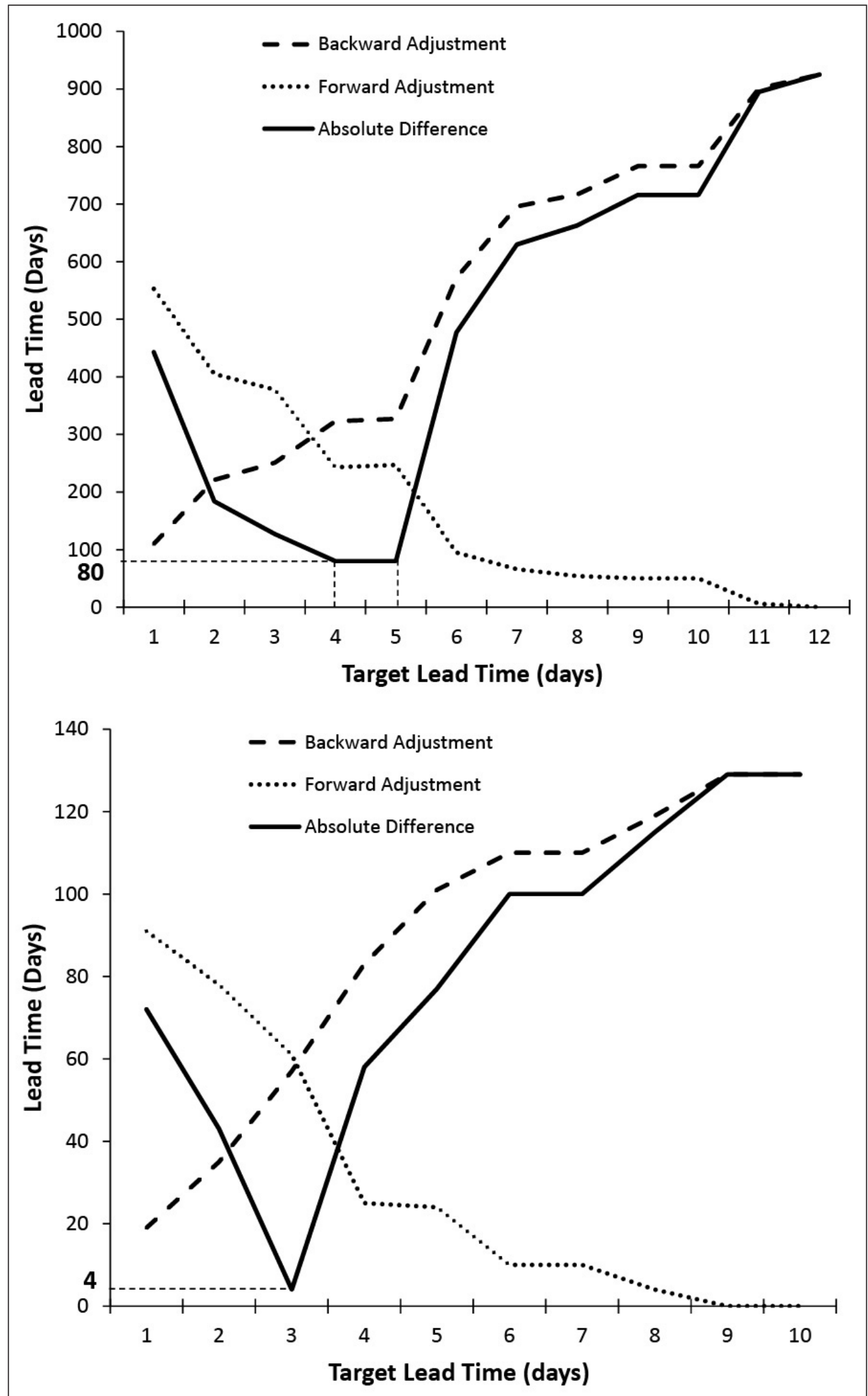

Fig. 1 Lead time optimization for a six-month appointment request for return visit patients (top) and for new patients (bottom) in a Pediatric clinic 


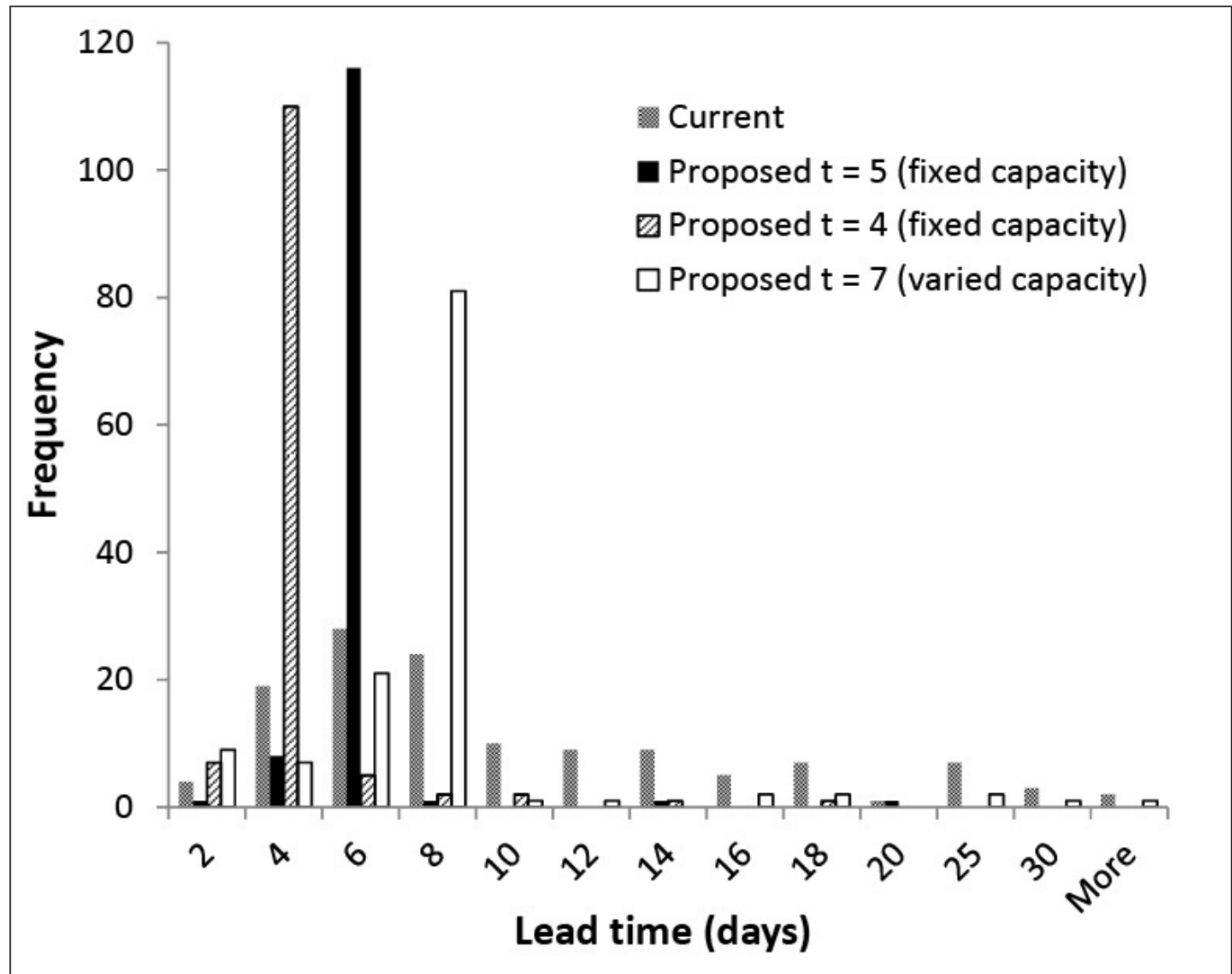

Fig. 2 Lead time comparison for a six-month (128 days) Pediatric RV appointment requests

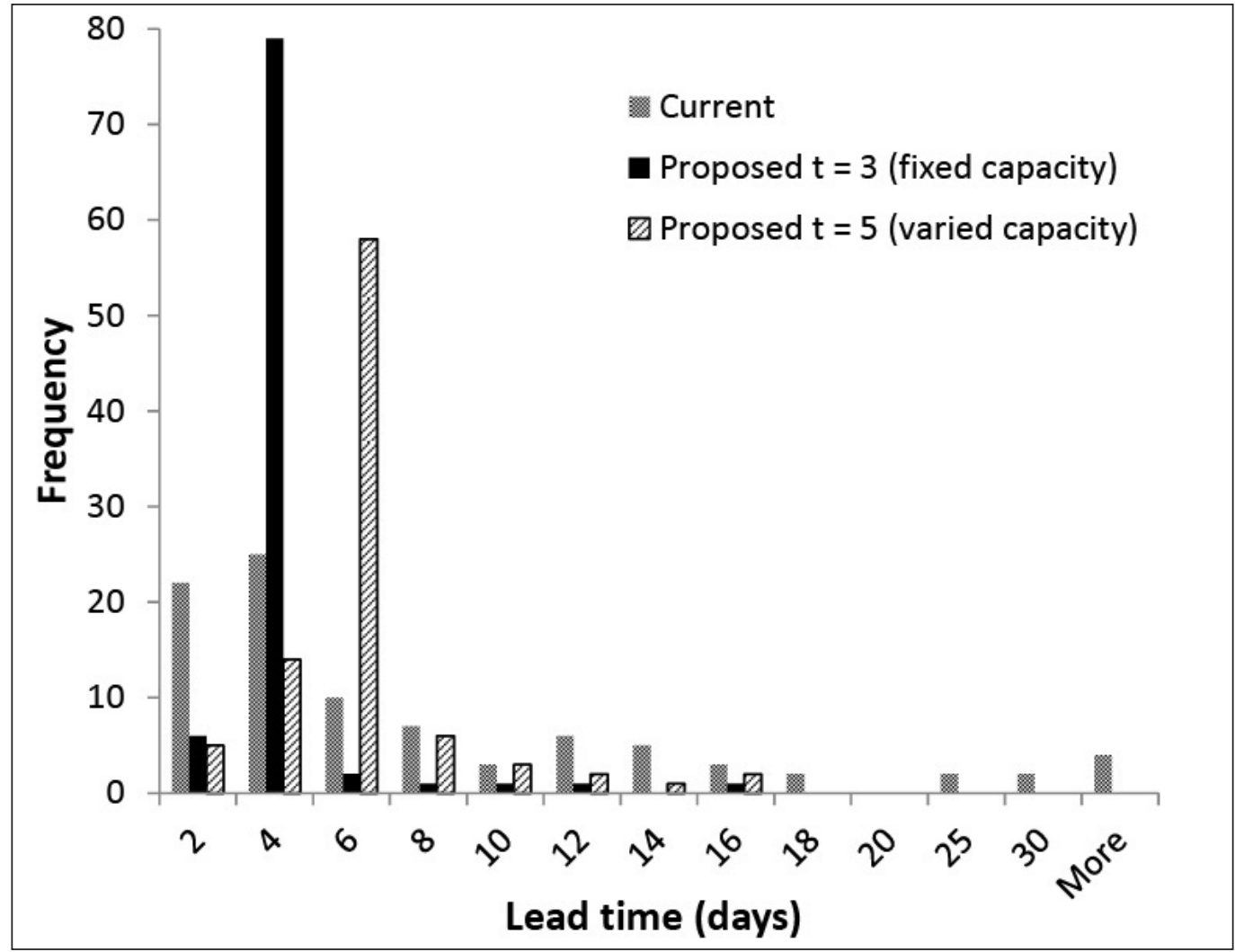

Fig. 3 Lead time comparison for a six-month (128 days) Pediatric NP appointment requests 


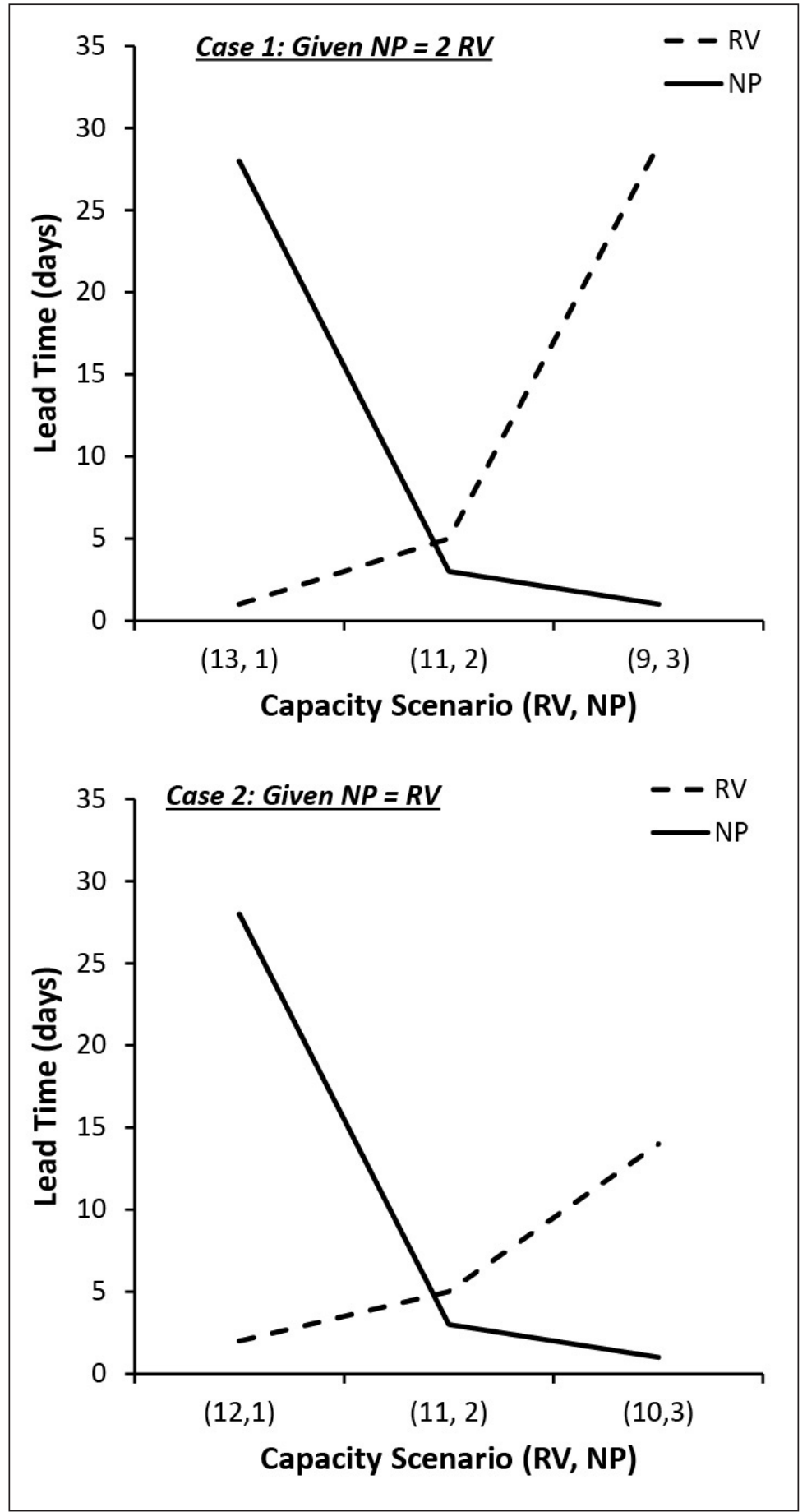

Fig. 4 The tradeoff of the target lead time when switching between NP and RV capacity 
Table 1 Example of Appointment Requests and Schedule Adjustments with 6-days of Requests and a Target Lead Time of Three Days $(t=3)$

\begin{tabular}{|c|c|c|c|c|c|c|c|c|c|c|c|c|}
\hline Day (i) & 1 & 2 & 3 & 4 & 5 & 6 & 7 & 8 & 9 & 10 & 11 & 12 \\
\hline Appointment request $R(i)$ & 5 & 6 & 3 & 7 & 2 & 9 & & & & & & \\
\hline $\begin{array}{l}\text { Schedule adj. to target lead time } \\
S_{t}(i)\end{array}$ & & & & 5 & 6 & 3 & 7 & 2 & 9 & & & \\
\hline Appointment capacity C(i) & 4 & 4 & 4 & 4 & 4 & 4 & 4 & 4 & 4 & 4 & 4 & 4 \\
\hline Excess cap. from target $E_{t}(i)$ & 4 & 4 & 4 & -1 & -2 & 1 & -3 & 2 & -5 & 4 & 4 & 4 \\
\hline \multicolumn{13}{|l|}{ Backward adjustment } \\
\hline \multicolumn{13}{|l|}{ Schedule adj. backward $S_{b}(i=9)$} \\
\hline move 2 from day $9 \rightarrow 8$ & & & & & & & & 4 & 7 & & & \\
\hline move 1 from day $9 \rightarrow 6$ & & & & & & 4 & & 4 & 6 & & & \\
\hline search stops at $i-t=6$ ( 2 moves) & 0 & 0 & 0 & 5 & 6 & 4 & 7 & 4 & 6 & 0 & 0 & 0 \\
\hline Excess cap. backward $E_{b}(i=9)$ & 4 & 4 & 4 & -1 & -2 & 0 & -3 & 0 & -2 & 4 & 4 & 4 \\
\hline \multicolumn{13}{|l|}{ Schedule adj. backward $S_{b}(i=7)$} \\
\hline search stops at $i-t=4$ (no move) & 0 & 0 & 0 & 5 & 6 & 4 & 7 & 4 & 6 & 0 & 0 & 0 \\
\hline Excess cap. backward $E_{b}(i=7)$ & 4 & 4 & 4 & -1 & -2 & 0 & -3 & 0 & -2 & 4 & 4 & 4 \\
\hline \multicolumn{13}{|l|}{ Schedule adj. backward $S_{b}(i=5)$} \\
\hline move 2 from day $5 \rightarrow 3$ & & & 2 & & 4 & & & & & & & \\
\hline search stops at $i-t=2$ (1 move) & 0 & 0 & 2 & 5 & 4 & 4 & 7 & 4 & 6 & 0 & 0 & 0 \\
\hline Excess cap. backward $E_{b}(i=5)$ & 4 & 4 & 2 & -1 & 0 & 0 & -3 & 0 & -2 & 4 & 4 & 4 \\
\hline \multicolumn{13}{|l|}{ Schedule adj. backward $S_{b}(i=4)$} \\
\hline move 1 from day $4 \rightarrow 3$ & & & 3 & 4 & & & & & & & & \\
\hline search stops at $i-t=1$ ( 1 move) & 0 & 0 & 3 & 4 & 4 & 4 & 7 & 4 & 6 & 0 & 0 & 0 \\
\hline Excess cap. backward $E_{b}(i=4)$ & 4 & 4 & 1 & 0 & 0 & 0 & -3 & 0 & -2 & 4 & 4 & 4 \\
\hline \multicolumn{13}{|l|}{ Forward adjustment } \\
\hline \multicolumn{13}{|l|}{ Schedule adj. forward $S_{f}(i=7)$} \\
\hline move 3 from day $7 \rightarrow 10$ & & & & & & & 4 & & & 3 & & \\
\hline search stops (1 move) & 0 & 0 & 3 & 4 & 4 & 4 & 4 & 4 & 6 & 3 & 0 & 0 \\
\hline Excess cap. forward $E_{f}(i=7)$ & 4 & 4 & 1 & 0 & 0 & 0 & 0 & 0 & -2 & 1 & 4 & 4 \\
\hline \multicolumn{13}{|l|}{ Schedule adj. forward $S_{f}(i=9)$} \\
\hline move 1 from day $9 \rightarrow 10$ & & & & & & & & & 5 & 4 & & \\
\hline move 1 from day $9 \rightarrow 11$ & & & & & & & & & 4 & 4 & 1 & \\
\hline search stops (2 moves) & 0 & 0 & 3 & 4 & 4 & 4 & 4 & 4 & 4 & 4 & 1 & 0 \\
\hline Excess cap. forward $E_{f}(i=9)$ & 4 & 4 & 1 & 0 & 0 & 0 & 0 & 0 & 0 & 0 & 3 & 4 \\
\hline
\end{tabular}


Table 2 Summary of Appointment Capacity Changes to Lead Time Policy

\begin{tabular}{|c|c|c|c|c|c|c|}
\hline Patient Type & $\begin{array}{l}\text { Appoint- } \\
\text { ment } \\
\text { Capacity }\end{array}$ & $\begin{array}{l}\text { Target } \\
\text { Lead } \\
\text { Time }\end{array}$ & $\begin{array}{l}\text { Backward } \\
\text { Adjustment }\end{array}$ & $\begin{array}{l}\text { Forward } \\
\text { Adjustment }\end{array}$ & $\begin{array}{l}\text { Absolute } \\
\text { Difference }\end{array}$ & $\begin{array}{l}\text { Average } \\
\text { Lead Time }\end{array}$ \\
\hline \multirow{6}{*}{$\begin{array}{l}\text { Return Visit } \\
\text { Patient (RV) }\end{array}$} & 13 & 1 & 86 & 71 & 15 & 1.0 \\
\hline & 12 & 2 & 182 & 122 & 60 & 1.1 \\
\hline & 11 & 4 & 323 & 243 & 80 & 3.9 \\
\hline & & 5 & 327 & 247 & 80 & 4.9 \\
\hline & 10 & 14 & 1935 & 2021 & 86 & 14.1 \\
\hline & 9 & 29 & 4484 & 4754 & 270 & 29.2 \\
\hline \multirow{3}{*}{$\begin{array}{l}\text { New Patient } \\
\text { (NP) }\end{array}$} & 3 & 1 & 10 & 5 & 5 & 1.0 \\
\hline & 2 & 3 & 57 & 61 & 4 & 3.0 \\
\hline & 1 & 28 & 1308 & 1242 & 66 & 28.6 \\
\hline
\end{tabular}




\section{References}

1. Norris S. The Wait Times Issue and The Patient Wait Times Guarantee. Social Affairs Division. Parliament of Canada. 2009.

2. Thomson S, Osborn R, Squires D, Jun M. International Profiles of Health Care Systems. The Commonwealth Fund. 2013.

3. Steedman NM, Worsnop V, Fernie R. Acceptance of genitourinary medicine clinic appointments within 48 hours is influenced by patient perception of risk but not by symptoms. Int J STD AIDS 2009; 20(9): 644-646.

4. Pacifico MD, Pearl RA, Grover R. The UK Government two-week rule and its impact on melanoma prognosis: an evidence-based study. Ann R Coll Surg Engl.2007; 89(6): 609-615.

5. UK Department of Health. The NHS Outcomes Framework 2014/2015. 2013.

6. Kaplan G, Lopez MH, McGinnis JM. Transforming Health Care Scheduling and Access: Getting to Now. Committee on Optimizing Scheduling in Heatlh Care; Institute of Medicine, editors. Washington, DC: The National Academies Press; 2015.

7. Dobson G, Hasija S, Pinker EJ. Reserving Capacity for Urgent Patients in Primary Care. Prod Oper Manag 2011; 20(3): 456-473.

8. Huang Y, Zuniga P, Marcak J. A cost-effective urgent care policy to improve patient access in a dynamic scheduled clinic setting. J Oper Res Soc 2014; 65(5): 763-776.

9. Qua X, Rardina RL, Williamsb JS, Willisc DR. Matching daily healthcare provider capacity to demand in advanced access scheduling systems. Eur J Oper Res 2007; 183(2): 812-826.

10. Harper PR, Gamlin HM. Reduced outpatient waiting times with improved appointment scheduling: a simulation modelling approach. OR Spectrum 2003: 25(2): 207-222.

11. Robinson LW, Chen RR. Scheduling doctors' appointments: optimal and empirically-based heuristic policies. IIE Trans 2003; 35(3): 295-307.

12. Huang Y, Hancock WM, Herrin GD. An alternative outpatient scheduling system: Improving the outpatient experience. IIE Trans Healthc Syst Eng 2012; 2(2): 97-111.

13. Qu X, Peng Y, Kong N, Shi J. A two-phase approach to scheduling multi-category outpatient appointments - a case study of a women's clinic. Health Care Manag Sci 2013; 16(3): 197-216.

14. Vanden Bosch PM, Dietz DC. Minimizing expected waiting in a medical appointment system. IIE Trans 2000; 32(9): 841-848.

15.Huang Y, Verduzco S. Appointment Template Redesign in a Women's Health Clinic Using Clinical Constraints to Improve Service Quality and Efficiency. Appl Clin Inform 2015; 6(2): 271-287.

16. Santibáñez P, Chow VS, French J, Puterman ML, Tyldesley S. Reducing patient wait times and improving resource utilization at British Columbia Cancer Agency's ambulatory care unit through simulation. Health Care Manag Sci 2009; 12(4): 392-407.

17. Kopach R, DeLaurentis PC, Lawley M, Muthuraman K, Ozsen L, Rardin R, Wan H, Intrevado P, Qu X, Willis D. Effects of clinical characteristics on successful open access scheduling. Health Care Manag Sci 2007; 10(2): 111-124.

18. Liu N, Ziya S, Kulkarni VG. Dynamic scheduling of outpatient appointments under patient no-Shows and cancellations. Manuf Serv Oper Manag 2010; 12(2): 347-364.

19. Huang Y, Hanauer DA. Patient No-Show Predictive Model Development using Multiple Data Sources for an Effective Overbooking Approach. Appl Clin Inform 2014; 5(3): 836-860.

20. Kim S, Giachetti RE. A Stochastic Mathematical Appointment Overbooking Model for Healthcare Providers to Improve Profits. IEEE Trans Syst Man Cybern 2006; 36(6): 1211-1219.

21. Huang Y, Zuniga P. Dynamic overbooking scheduling system to improve patient access. J Oper Res Soc 2012; 63(6): 810-820.

22. Robinson LW, Chen RR. A Comparison of Traditional and Open-Access Policies for Appointment Scheduling. Manuf Serv Oper Manag 2010; 12(2): 330-346.

23. Murray $\mathrm{M}$, Bodenheimer $\mathrm{T}$, Rittenhouse $\mathrm{D}$, Grumbach $\mathrm{K}$. Improving timely access to primary care: case studies of the advanced access model. JAMA 2003; 289(8): 1042-1046.

24. Mehrotra A, Keehl-Markowitz L, Ayanian JZ. Implementing open-access scheduling of visits in primary care practices: a cautionary tale. Ann Intern Med 2008; 148(12): 915-922.

25. Silvester KLR, Richard L, Bevan H, Steyn RS, Walley P. Reducing waiting times in the NHS: is lack of capacity the problem? Clinician Manag 2004; 12(3): 105-111.

26. Patrick J. A Markov decision model for determining optimal outpatient scheduling. Health Care Manag Sci 2012; 15(2): 91-102.

27.Gocgun Y, Puterman ML. Dynamic scheduling with due dates and time windows: an application to chemotherapy patient appointment booking. Health Care Manag Sci 2014; 17(1): 60-76. 
28. Matteson-Kome ML, Lopez KT, Sliger AD, Mathews MJ, Bechtold ML. Improving care access for new patients in an outpatient gastroenterology clinic: a novel approach. Mo Med 2014; 111(6): 512-515.

29. Snow BW, Cartwright PC, Everitt S, Ekins M, Maudsley W, Aloi S. A method to improve patient access in urological practice. J Urol 2009; 182(2): 663-667.

30. O'Neill S, Calderon S, Casella J, Wood E, Carvelli-Sheehan J, Zeidel ML. Improving outpatient access and patient experiences in academic ambulatory care. Acad Med 2012; 87(2): 194-199.

31.Hanning M, Spangberg UW. Maximum wait time - a threat to clinical freedom? Implementation of a policy to reduce waiting times. Health Policy 2000; 52(1): 15-32.

32. Calltorp J. Priority setting in health policy in Sweden and a comparison with Norway. Health Policy 1999; 50(1-2): 1-22.

33. Hole AR. Modelling heterogeneity in patients' preferences for the attributes of a general practitioner appointment. J Health Econ 2008; 27(4): 1078-1094.

34.Dexter F, Macario A, Traub RD, Hopwood M, Lubarsky A. An Operating Room Scheduling Strategy to Maximize the Use of Operating Room Block Time: Computer Simulation of Patient Scheduling and Survey of Patients' Preferences for Surgical Waiting Time. Anesth Analg 1999; 89(1): 7-20.

35. Conner-Spady BL, Sanmartin C, Johnston GH, McGurran JJ, Kehler M, Noseworthy TW. The importance of patient expectations as a determinant of satisfaction with waiting times for hip and knee replacement surgery. Health Policy 2011; 101(3): 245-252.

36. Rubin G, Bate A, George A, Shackley P, Hall N. Preferences for access to the GP: a discrete choice experiment. Brit J Gen Pract 2006; 56(531): 743-748.

37. Turner D, Tarrant C, Windridge K, bryan S, Boulton M, Freeman G, Baker R. Do patients value continuity of care in general practice? An investigation using stated preference discrete choice experiments. J Health Serv Res Policy 2007; 12(3): 132-137. 